To be presented at Microscopy \& Microanalysis 98

Atlanta, Georgia, July 12-16, 1998, and published in Proceedings

\title{
Investigation of the Local Superconducting Properties at Grain Boundaries in High- $T_{c}$ Superconductors
}

C. Prouteau, ${ }^{1,2}$ G. Duscher, ${ }^{1,2}$ N. D. Browning, ${ }^{2}$ S. J. Pennycook, ${ }^{1}$

D. Verebelyi, ${ }^{1}$ D. K. Christen, ${ }^{1}$ M. F. Chisholm, ${ }^{1}$ and D. P. Norton ${ }^{1}$

1Solid State Division, Oak Ridge National Laboratory

P.O. Box 2008, Oak Ridge, Tennessee 37831-6030

2Department of Physics, University of Illinois at Chicago Chicago, Illinois 60607-7059

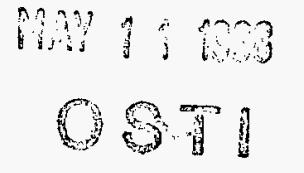

The submitted manuscript bas been authored by a contractor of the U.S. Government under coniract No. DE-AC05-960R22464. Aconting Accordingly, the U.S. Government retains a nonexclusive, royalty-free license to publish or reproduce the published form of this contribution, or allow others to do so, for U.S. Government purposes.

DISTAIBUTION OF THS DOOMENT IS UNLIMTEE
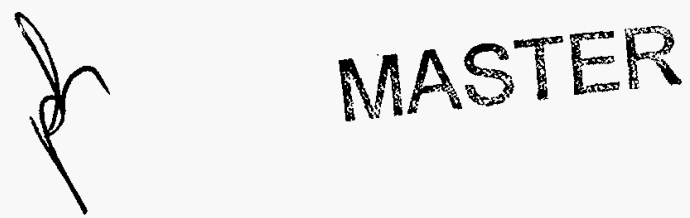

\author{
prepared by \\ SOLID STATE DIVISION \\ OAK RIDGE NATIONAL LABORATORY \\ Managed by \\ LOCKHEED MARTIN ENERGY RESEARCH CORP. \\ under \\ Contract No. DE-AC05-96OR22464 \\ with the
}

U.S. DEPARTMENT OF ENERGY

Oak Ridge, Tennessee

February 1998
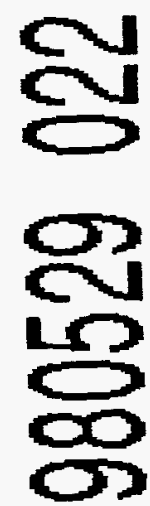


\section{DISCLAIMER}

This report was prepared as an account of work sponsored by an agency of the United States Government. Neither the United States Government nor any agency thereof, nor any of their employees, makes any warranty, express or implied, or assumes any legal liability or responsibility for the accuracy, completeness, or usefulness of any information, apparatus, product, or process disclosed, or represents that its use would not infringe privately owned rights. Reference herein to any specific commercial product, process, or service by trade name, trademark, manufacturer, or otherwise does not necessarily constitute or imply its endorsement, recommendation, or favoring by the United States Government or any agency thereof. The views and opinions of authors expressed herein do not necessarily state or reflect those of the United States Government or any agency thereof. 


\title{
INVESTIGATION OF THE LOCAL SUPERCONDUCTING PROPERTIES AT GRAIN BOUNDARIES IN HIGH-TC SUPERCONDUCTORS
}

\author{
C. Prouteau *\#, G. Duscher*\#, N. D. Browning\#, S. J. Pennycook*, D. Verebelyi*, \\ D. K. Christen*, M. F. Chisholm*, D. P. Norton* \\ * Solid State Division, Oak Ridge National Laboratory, Oak Ridge, TN 37381-6031, USA \\ \# Department of Physics, University of Illinois at Chicago, Chicago. Il 60607-7059, USA.
}

Developing an atomic scale study of the structure-property relationships of grain boundaries in high- $T_{c}$ superconductors is essential to understand their current dissipation mechanism and for incorporating these materials into viable devices. Thin $\mathrm{YBa}_{2} \mathrm{Cu}_{3} \mathrm{O}_{7 . \delta}$ films have been deposited by pulsed laser deposition (PLD) on $\mathrm{SrTiO}_{3}$ symmetric bicrystals. Transport measurements in a magnetic field have been conducted across the grain boundaries through a wide bridge. The data obtained are consistent with microstructural observation in a VG Microscopes HB603 U and a VG HB501 UX dedicated STEM. Of particular interest in the study of high- $T_{c}$ materials is the use of EELS, which can highlight the presence of non-superconducting regions through interpretation of the onset positions and finestructure (ELNES) of characteristic core-edge features 1.2 .3 .

The V(I) curves recorded across a $24^{\circ}$ boundary for several magnetic fields (fig. 1 - left) show an onset critical current density followed by a linear differential ohmic behavior which gives a negative intercept. The small $\mathrm{J}_{c}(B)$ arises from a small fraction of strong links, the linear differential resistivity regime represents the grain boundary resistance, and the rapid rise at high current densities is due to the properties of the grains. To explain these properties, a model, where the grain boundary is composed of equally sized and spaced dislocation cores separated by a very small fraction of much stronger links which might carry either the depairing current or the $J_{c}$ of the grains or a stronger Josephson current is shown in the center of fig. 1. The depairing current case seems very unlikely due to the observed field dependence. The simulated graph obtained for this model, for a situation where the area fraction $x=0.005$, and $J_{c}$ is the observed $J_{c}(B)$ of the grains (fig. 1 - right), exhibits qualitatively similar behavior to the data on the left, (right). The imperfect fit suggests that the exact nature of these strong links is most likely a much stronger Josephson current.

The model proposed from the transport properties data is consistent with Z-contrast image of a $30^{\circ}$ grain boundary (fig. 2 ) which shows an asymmetric segment on the left, containing a slightly distorted unit cell, and a small symmetric segment on the right. According to EELS measurements at such boundaries, the symmetry of the boundary seems to play an important role in determining superconducting properties. The ELNES oxygen $\mathrm{K}$ edge has been recorded across symmetric and asymmetric boundaries (fig. 3 ). The intensity of the oxygen $\mathrm{K}$ pre-edge which is related to the hole concentration doesn't change at symmetric boundaries but disappears at asymmetric boundaries. This result suggest that the presence of a very small fraction of symmetric cells in a predominantly asymmetric boundary might be the stronger links of the model proposed.

This preliminary investigation has shown that the study of the grain boundary electronic microstructure in parallel with transport properties study should lead us to a better understanding of the transport properties of the superconducting materials. In particular, it has been shown that transport measurements are consistent with a model were the grain boundaries are composed of weak links separated by a very small fraction of much stronger links although more boundaries need to be studied to draw definitive conclusions and determine the real nature of these strong links.

\footnotetext{
References

${ }^{1}$ N.D.Browning \& al. Interface Science 1, 309 (1993)

${ }^{2}$ J.Fink \& al. Phys. Rev. B 42, 4823 (1990)

${ }^{3}$ N.Nucker \& al. Phys. Rev. B 39, 6619 (1989)

${ }^{4}$ We would like to thank P. D. Nellist for the image presented in fig. 2 and J. T. Luck for her help with sample preparation. This program is supported by NSF under grant no. DMR-9503877, and by the Division of Materials Sciences, U.S. DOE, under contract DE-AC05-96OR22464 with Lockheed Martin Energy Research Corporation, and by appointment to the ORNL Postdoctoral Research Program administered jointly by ORNL and ORISE.
} 
1.
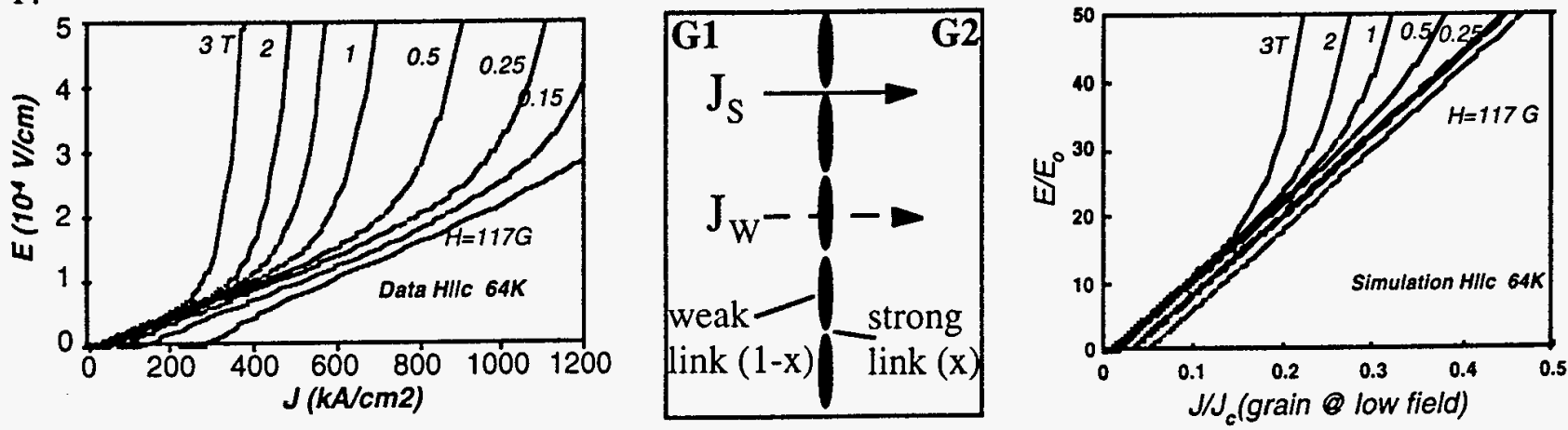

2.

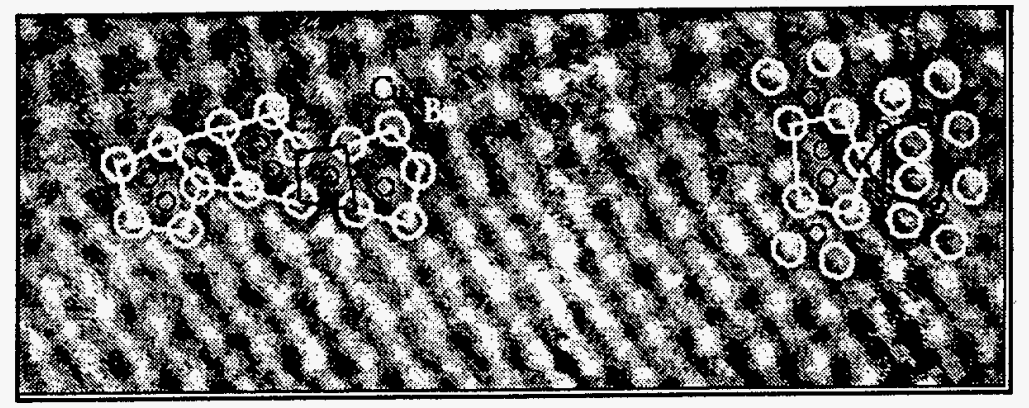

3.
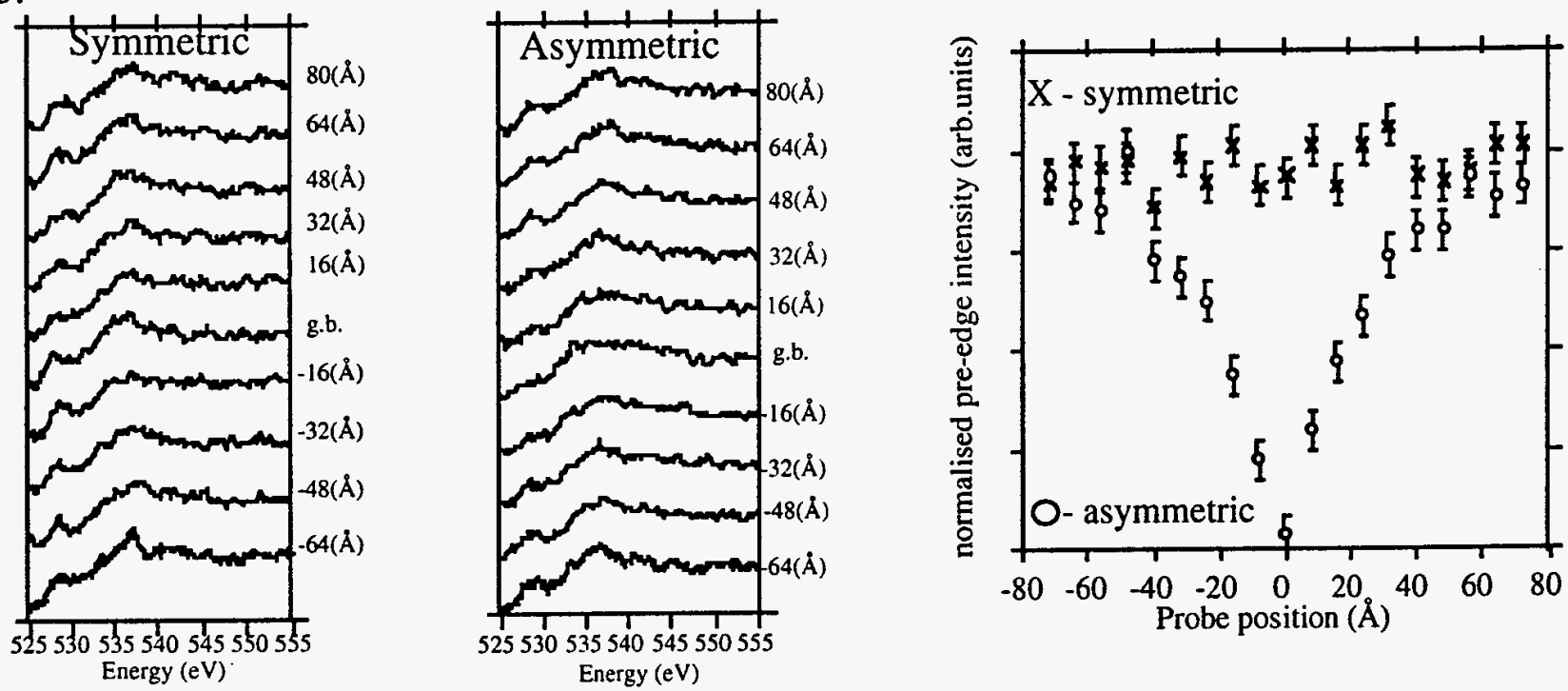

FIG. 1. (left) V(I) curves for several magnetic fields. The small $J_{c}(B)$ arises from the strong links, the linear differential resistivity regime represents the grain boundary resistance, and the rapid rise at high current densities is due to the properties of the grains. (center) In the proposed model, the grain boundary is composed of equally sized and spaced dislocation cores separated by a very small fraction of strong links. (right) The simulated graph exhibits qualitatively similar behavior to the data on the left, for a situation where the fraction $x=0.005$, and $J_{c}$ is the observed $J_{c}(B)$ of the grains.

FIG. 2. Z-contrast image of a $30^{\circ}$ grain boundary showing an asymmetric segment on the left, containing a distorted unit cell, and a small symmetric segment on the right.

FIG. 3. EELS oxygen $K$ edge across boundaries. The hole concentration profile obtained from the pre-edge shows a radically different behavior between asymmetric and symmetric boundaries. It disappears at the asymmetric boundary. 


\section{M98004939}

||||||||||||||||||||||||||||||||||||||||||||||||||||||||

Report Number (14) $\frac{3 P N L / C P--96835}{C O N F-980713-\cdots}$

Publ. Date (11) DoE 199802
Sponsor Code (18)/ER, XF
UC Category (19) $U C-4 C A, D O E / E R$ 